\title{
Hospital costs for patients with lower extremity cellulitis: a retrospective population-based study
}

\author{
Douglas Challener ${ }^{1}$, Jasmine Marcelin ${ }^{2}$, Sue Visscher ${ }^{1}$, and Larry Baddour ${ }^{1}$ \\ ${ }^{1}$ Mayo Clinic - Internal Medicine, 200 1st St SW, Rochester, Minnesota 55901, United States \\ ${ }^{2}$ University of Nebraska Medical Center, Omaha, Nebraska, United States
}

\begin{abstract}
Background-Hospital admissions for non-purulent lower extremity cellulitis (NLEC) are common and can be prolonged and costly. Newer treatment options and preventive strategies are expected to result in cost savings before implementation, but few studies have quantified the cost of conventional treatment.
\end{abstract}

Methods-Using the Rochester Epidemiology Project, the incidence of NLEC in Olmsted County, MN in 2013 was 176.6 per 100,000 persons. The subset of patients who required hospitalization for NLEC in 2013 was determined. Hospital admissions were analyzed retrospectively using standardized cost analysis within several relevant categories.

Results-Thirty-four patients had an average hospital length of stay of 4.7 days. The median total inpatient cost was $\$ 7,341$. The median cost per day was $\$ 2,087$, with $49 \%$ due to room and board. Antibiotics administered for treatment of NLEC contributed a median cost of $\$ 75$ per day of hospitalization, and laboratory and imaging test costs were $\$ 73$ and $\$ 44$, respectively, per day of hospitalization.

Conclusions-Hospitalizations for NLEC can be costly and prolonged with room and board accounting for much of the cost. Therefore, newer management strategies should seek to reduce hospital length of stay and/or avoid inpatient admission to reduce cost.

\section{Keywords}

Lower extremity cellulitis; cost; hospitalization; population-based; Rochester Epidemiology Project; inpatient

\section{Introduction}

Non-purulent lower extremity cellulitis (NLEC) is commonly seen in both inpatient and outpatient settings. Several studies have defined the incidence of NLEC, but few have attempted to determine the cost of inpatient management of this infection. The incidence of NLEC was approximately 199 per 100,000 person-years in the Olmsted County, Minnesota

\footnotetext{
Declarations of Interests

The authors have no relevant affiliations or financial involvement with any organization or entity with a financial interest in or financial conflict with the subject matter or materials discussed in the manuscript. This includes employment, consultancies, honoraria, stock ownership or options, expert testimony, grants or patents received or pending, or royalties.
} 
population in 1999 with about 80 percent of the diagnoses made in the outpatient setting.[1] The NLEC incidence in this population was re-examined in the 2013 population after the emergence of community-acquired methicillin-resistant Staphylococcus aureus (CAMRSA), with a decreased incidence of 176.6 per 100,000.[2] Despite evidence of a diminishing incidence of NLEC in our local population and in others[3], the prevalence of hospitalization among the local population remained stable (approximately 20\%) for both cohorts.[1,2] While the majority of care for NLEC occurs in ambulatory settings, the bulk of the overall costs are incurred in the hospital.[4] Inpatient treatment is recommended for moderate and severe skin and soft tissue infections, as well as for patients who are severely immunocompromised, may be poorly compliant, or those who have failed outpatient treatment.[5] The presence of other comorbidities and other active medical problems may also impact the decision to admit to the hospital.[6]

Determining inpatient costs of NLEC is important as new treatment strategies are implemented for more efficient care. Outpatient infusion centers have been popularized recently and have been cost effective for intravenous antibiotic administration when compared to inpatient admissions.[7] Similarly, home administration of intravenous antibiotics via home healthcare agencies aims to reduce costs. Moreover, prophylactic antibiotics have been effective in preventing NLEC recurrence, $[8,9]$ which commonly occurs in patients with a first episode of NLEC; additionally, prophylaxis can be cost effective, even after the first episode of NLEC.[10] Recently introduced antibiotics, including the long half-life lipoglycopeptides (oritavancin, telavancin and dalbavancin) also provide opportunities for effective treatment with the potential to avoid hospitalization and outpatient parenteral antibiotic therapy (OPAT) costs. In addition, "automatic" Infectious Disease consultation can improve outcome of NLEC patients diagnosed in the emergency department, by identifying cellulitis mimics[11, 12] (and avoiding antimicrobials), preventing hospitalization, and reducing recurrent episodes.[13]

NLEC is a significant burden on the healthcare system. With many treatment and prevention strategies that have been developed recently, it is important to determine the contemporary financial burden of treatment of NLEC. Quantifying the extent of that burden will assist in comparing new treatment or prevention strategies to current approaches. In this populationbased study, costs associated with inpatient admission for NLEC were quantified by evaluating a regional cohort seen in 2013. This is the first population-based study to define the cost of hospital treatment of NLEC in the United States.

\section{Methods}

Investigators defined the incidence of NLEC in Olmsted County, MN with use of the Rochester Epidemiology Project (REP), a unique research infrastructure that links together nearly all of the medical records of the residents of Olmsted County, MN.[14] Cases were found by identifying potential encounters with relevant ICD-9 codes for cellulitis and abscess, excluding encounters utilizing procedural codes for abscess drainage (Supplementary Tables 1 and 2) and finally confirmed via manual review of the electronic medical records. Data from the year 2013 were examined to identify patients requiring hospitalization. Standardized costs for the patients' billed services while in the hospital were 
obtained from the Rochester Epidemiology Project Cost Data Warehouse[15] which applies Medicare reimbursement to professional services and multiplies service line hospital charges by Medicare cost report cost-to-charge ratios. Costs are reported in 2013 US dollars.

Examined costs included total hospitalization cost and hospitalization cost per day, relevant laboratory cost per day, relevant imaging cost per day, and antibiotic cost per day. Laboratory tests included complete blood count, C-reactive protein (CRP) and erythrocyte sedimentation rate (ESR), basic chemistry panels, streptococcal serologies, including antistreptolysin O (ASO) and DNAse B titers, and blood cultures. Relevant imaging tests included lower extremity ultrasound, lower extremity plain radiography, and advanced imaging including computed tomographic scanning and magnetic resonance imaging). Relevant antibiotics included all oral, intravenous, and intramuscular antibiotics. Oral antibiotics included in the analysis were cephalexin, cefadroxil, levofloxacin, ciprofloxacin, moxifloxacin, clindamycin, dicloxacillin, penicillin VK, doxycycline, minocycline, metronidazole, amoxicillin-clavulanate, trimethoprim-sulfamethaxazole, linezolide, and amoxicillin. Intravenous antibiotics included ceftriaxone, cefazolin, cefepime, vancomycin, nafcillin, piperacillin/tazobactam, levofloxacin, ciprofloxacin, ertapenem, clindamycin, penicillin $\mathrm{G}$ potassium, aztreonam, linezolid, and ampicillin-sulbactam. The analysis also included the use of intramuscular ceftriaxone.

\section{Results}

Of 195 patients with confirmed NLEC episodes in the 2013 Olmsted County cohort, 34 required inpatient admission. The average LOS was 4.7 days (median 3.5 days; range, 1-16 days). Prolonged stay (hospitalization of more than five days[16]) occurred in 8 of the 34 inpatient admissions (33\%). The median total standardized inpatient cost was $\$ 7,341$. The median total cost per day was $\$ 2,087$. Median daily laboratory costs were $\$ 73$.

Twenty-six (76\%) patients underwent imaging of the lower extremity. Median daily imaging costs related to the lower extremity were $\$ 44$. The most frequent imaging study was duplex ultrasound scanning of the lower extremity vasculature (26 [43\%] of 60 total imaging studies). The remainder included a variety of plain radiographs of the lower extremity. No patient underwent CT or MRI of the lower extremity. Patients who had lower extremity imaging had a mean total cost per day of $\$ 2,306$ as compared to a mean total cost per day of $\$ 1,796$ for those without imaging. Patients without lower extremity imaging also had a shorter average length of stay (3.25 days; 95\% 2.27-4.22; vs. 5.11 days; 95\% 3.48-6.74).

Median daily antibiotic costs related to NLEC were $\$ 75$ (Table 1). The vast majority of antibiotics were administered intravenously with only $26 \%$ of antibiotic doses being given orally (most commonly cephalexin). The most commonly administered antibiotic was intravenous vancomycin at $21 \%$ of administered doses. Both intravenous cefazolin and piperacillin-tazobactam were also commonly provided with $13 \%$ and $8 \%$ of administered doses respectively.

Total standardised costs varied widely from $\$ 4,313$ at the $25^{\text {th }}$ percentile to $\$ 11,733$ at the $75^{\text {th }}$ percentile. When normalized for the length of stay, data became more uniform with a 
mean total cost per day of $\$ 2,186,25^{\text {th }}$ percentile of $\$ 1,773$ and $75^{\text {th }}$ percentile of $\$ 2,574$ (Table 1).

The cohort was divided into two groups, based on length of stay. Patients who were in the hospital more than 5 days had a mean cost per day of $\$ 2,637$ (95\%: $\$ 1,770-\$ 3,504)$. The patients who were in the hospital for shorter stays had lower costs even when normalized for length of stay. These patients had a mean cost per day of $\$ 2,047$ (95\%: $\$ 1,839$ to $\$ 2,256$ ).

Six inpatients underwent procedures which contributed to the total cost of their hospitalizations. The patients who received procedures while inpatient had an average cost per day of $\$ 3,033$ (95\%: $\$ 1,968-\$ 4,097)$. Those who did not have procedures had an average cost per day of $\$ 2,005$ (95\%: $\$ 1,822-\$ 2,187)$. The patient with the highest total hospital cost $(\$ 66,669)$ also had the second longest length of stay at 14 days. The procedure costs for this patient were the highest in the cohort at a total of $\$ 6,734$. This hospital stay was complicated by Staphylococcus aureus blood stream infection and septic joints requiring multiple joint aspirations and debridement operations contributing to the high procedural costs. None of the patients who underwent inpatient procedures were hospitalized with the primary diagnosis of NLEC. All had procedures relating to their other diagnoses including closed reduction of fibular fracture, colonoscopic decompression, lumbar puncture and paracentesis.

Overall, the largest component of the hospital cost was room and board which accounted for, on average, $49 \%$ of the total cost with an average cost per day for room and board of $\$ 1045$.

\section{Discussion}

This is the first population-based investigation to evaluate the costs of inpatient treatment of NLEC in a well-defined, contemporary, American population. This study showed that inpatient treatment of cellulitis was costly, with the largest proportion of the cost due to daily room and board. The average cost per day increased as the LOS increased.

Published data that examine hospitalization for NLEC is sparse, although there have been several international attempts at quantifying the inpatient costs of NLEC. These have ranged in average length-of-stay (LOS) from 5.8 to 12.1 and total costs of 5346 Euro and AUS $\$ 5,196 .[4,17]$ A recent American database analysis showed median cost of hospitalization for cellulitis (not limited to NLEC) of \$5,159 with median LOS of 2.88 days.[18] An American study of diabetic patients hospitalized with skin/soft tissue infections (SSTIs) had an average LOS of 7.8 days with an average cost of $\$ 10,946$ per hospitalization.[19] One previous attempt at quantifying the cost of hospitalization associated with SSTIs in diabetics estimated that soft tissue infection contributed approximately $\$ 10,000$ per hospitalization to the total charge.[17]

The average LOS in the current study was similar to that for other studies (between 5 and 8 days).[20, 21] Interventions, therefore, that limit or prevent recurrence of NLEC may be important in preventing hospitalization. 
Expert opinion and published data suggest that prophylactic oral antibiotics reduce recurrences of NLEC.[5, 9] If the consistent use of prophylactic antibiotics results in fewer hospitalizations and shorter hospital stays, then they could have a significant impact on reducing the cost of NLEC. This has been suggested in cost analyses of the UK randomized controlled trials (PATCH I and II), which demonstrated that antibiotic prophylaxis is at least $62 \%$ cost effective to both the healthcare system and society.[8] Risk factor modification including treatment of lymphedema and tinea pedis is also recommended for reduction of cellulitis recurrence.[22]

Current inpatient costs related to antibiotics are relatively low compared to the proportion of costs from other management categories. The most effective strategy for reducing costs may be to simply treat patients without admission to the hospital with the use of outpatient infusion centers for intravenous antibiotics, if needed. This may be a more cost effective option than the use of the novel long half-life lipoglycopeptides which are currently very costly and achieve the same goal of avoiding inpatient admission. In general, outpatient parenteral antibiotic delivery is cost-effective and significantly less expensive than inpatient delivery of the same agents.[23, 24] In addition, patients who receive antibiotics through an OPAT program receive antibiotics for a shorter duration than those who remained hospitalized.[7] If OPAT therapy is selected, then early close outpatient follow-up will be needed.

This retrospective study is limited by the relatively small cohort of inpatient admissions for NLEC in this population in one year. Only 34 patients of 195 with confirmed NLEC diagnoses required hospitalization. The small sample size may limit the generalizability of these results. Additionally, the study likely underestimated the financial burden of NLEC as the vast majority of patients are treated without hospitalization and many may never seek healthcare. The retrospective nature of the study forces reliance on the accuracy of documentation rather than allowing the investigators to confirm diagnosis of NLEC in person. Coding practices differ across hospitals which may also lead to failure to capture all cases of NLEC. The relatively rural, Midwestern setting for this study likely has effects on costs as well as other confounding factors such as average length of stay. The study benefits from being population-based, including data from all the hospitals within the county.

\section{Conclusions}

This study demonstrated the standardized costs involved in the inpatient treatment of NLEC in a geographically-defined cohort in the Upper Midwest. The highest proportion of costs was not related to medications, interventions, or testing but rather miscellaneous charges related to inpatient admission, especially room and board. Cost-effective treatment of this disease should focus on prevention of NLEC recurrence, which is common in NLEC, and treatment strategies that include detailed ambulatory care management for the portion of patients who otherwise can be safely managed in this way.

\section{Supplementary Material}

Refer to Web version on PubMed Central for supplementary material. 


\section{Acknowledgments}

\section{Funding}

This publication was supported by the Mayo Foundation for Research and Education through the Division of Infectious Diseases, by the Rochester Epidemiology Project (Grant number R01-AG034676; Principal Investigators: Walter A. Rocca, MD, and Barbara P. Yawn, MD, MSc), by funding from the Mayo Clinic Robert D. and Patricia E. Kern Center for the Science of Health Care Delivery and by Grant Number UL1 TR000135 from the National Center for Advancing Translational Sciences (NCATS). Its contents are solely the responsibility of the authors and do not necessarily represent the official views of the NIH.

\section{List of abbreviations}

NLEC Non-purulent lower extremity cellulitis

SSTI Skin and soft-tissue infections

LOS Length of stay

OPAT Outpatient parenteral antimicrobial therapy

REP Rochester Epidemiology Project

\section{References}

1. McNamara DR, et al. Incidence of lower-extremity cellulitis: a population-based study in Olmsted county, Minnesota. Mayo Clin Proc. 2007; 82(7):817-21. [PubMed: 17605961]

2. Marcelin JR, et al. Incidence and Effects of Seasonality on Nonpurulent Lower Extremity Cellulitis After the Emergence of Community-Acquired Methicillin-Resistant Staphylococcus aureus. Mayo Clin Proc. 2017

3. Morgan E, Daum RS, David MZ. Decreasing Incidence of Skin and Soft Tissue Infections With a Seasonal Pattern at an Academic Medical Center, 2006-2014. Open Forum Infectious Diseases. 2016; 3(4):ofw179. [PubMed: 28852669]

4. Kameshwar K, et al. False economies in home-based parenteral antibiotic treatment: a healtheconomic case study of management of lower-limb cellulitis in Australia. J Antimicrob Chemother. 2016; 71(3):830-5. [PubMed: 26702920]

5. Stevens DL, et al. Practice guidelines for the diagnosis and management of skin and soft tissue infections: 2014 update by the infectious diseases society of America. Clin Infect Dis. 2014; 59(2): 147-59. [PubMed: 24947530]

6. Pollack CV Jr, et al. Acute bacterial skin and skin structure infections (ABSSSI): practice guidelines for management and care transitions in the emergency department and hospital. J Emerg Med. 2015; 48(4):508-19. [PubMed: 25605319]

7. Chapman ALN, et al. Clinical efficacy and cost-effectiveness of outpatient parenteral antibiotic therapy (OPAT): a UK perspective. Journal of Antimicrobial Chemotherapy. 2009; 64(6):1316-24. [PubMed: 19767623]

8. Mason JM, et al. Prophylactic antibiotics to prevent cellulitis of the leg: economic analysis of the PATCH I \& II trials. PLoS One. 2014; 9(2):e82694. [PubMed: 24551029]

9. Oh CC, et al. Antibiotic prophylaxis for preventing recurrent cellulitis: a systematic review and meta-analysis. J Infect. 2014; 69(1):26-34. [PubMed: 24576824]

10. Thomas KS, et al. Penicillin to prevent recurrent leg cellulitis. N Engl J Med. 2013; 368(18):1695703. [PubMed: 23635049]

11. Hirschmann JV, Raugi GJ. Lower limb cellulitis and its mimics: part I. Lower limb cellulitis. J Am Acad Dermatol. 2012; 67(2):163 e1-12. quiz 175-6. [PubMed: 22794815]

12. Weng QY, et al. Costs and Consequences Associated With Misdiagnosed Lower Extremity Cellulitis. JAMA Dermatol. 2016 
13. Jain SR, et al. Infectious diseases specialist management improves outcomes for outpatients diagnosed with cellulitis in the emergency department: a double cohort study. Diagn Microbiol Infect Dis. 2017; 87(4):371-375. [PubMed: 28162827]

14. St Sauver JL, et al. Use of a medical records linkage system to enumerate a dynamic population over time: the Rochester epidemiology project. Am J Epidemiol. 2011; 173(9):1059-68. [PubMed: 21430193]

15. Visscher SL, et al. Developing a standardized healthcare cost data warehouse. BMC Health Serv Res. 2017; 17(1):396. [PubMed: 28606088]

16. Garg A, et al. Clinical characteristics associated with days to discharge among patients admitted with a primary diagnosis of lower limb cellulitis. J Am Acad Dermatol. 2017; 76(4):626-631. [PubMed: 28089727]

17. Hatoum HT, Akhras KS, Lin SJ. The attributable clinical and economic burden of skin and skin structure infections in hospitalized patients: a matched cohort study. Diagn Microbiol Infect Dis. 2009; 64(3):305-10. [PubMed: 19501792]

18. Peterson RA, et al. Increasing Incidence, Cost, and Seasonality in Patients Hospitalized for Cellulitis. Open Forum Infect Dis. 2017; 4(1):ofx008. [PubMed: 28480281]

19. Lipsky BA, et al. Skin and soft tissue infections in hospitalised patients with diabetes: culture isolates and risk factors associated with mortality, length of stay and cost. Diabetologia. 2010; 53(5):914-23. [PubMed: 20146051]

20. Ronnen M, et al. Erysipelas. Changing faces. Int J Dermatol. 1985; 24(3):169-72. [PubMed: 3997339]

21. Jorup-Ronstrom C, et al. The course, costs and complications of oral versus intravenous penicillin therapy of erysipelas. Infection. 1984; 12(6):390-4. [PubMed: 6394505]

22. Dalal A, et al. Interventions for the prevention of recurrent erysipelas and cellulitis. The Cochrane database of systematic reviews. 2017:6.

23. Dalovisio JR, et al. Financial impact of a home intravenous antibiotic program on a medicare managed care program. Clinical Infectious Diseases. 2000; 30(4):639-42. [PubMed: 10770722]

24. Wai AO, et al. Cost analysis of an adult outpatient parenteral antibiotic therapy (OPAT) programme. A Canadian teaching hospital and Ministry of Health perspective. Pharmacoeconomics. 2000; 18(5):451-7. [PubMed: 11151398] 


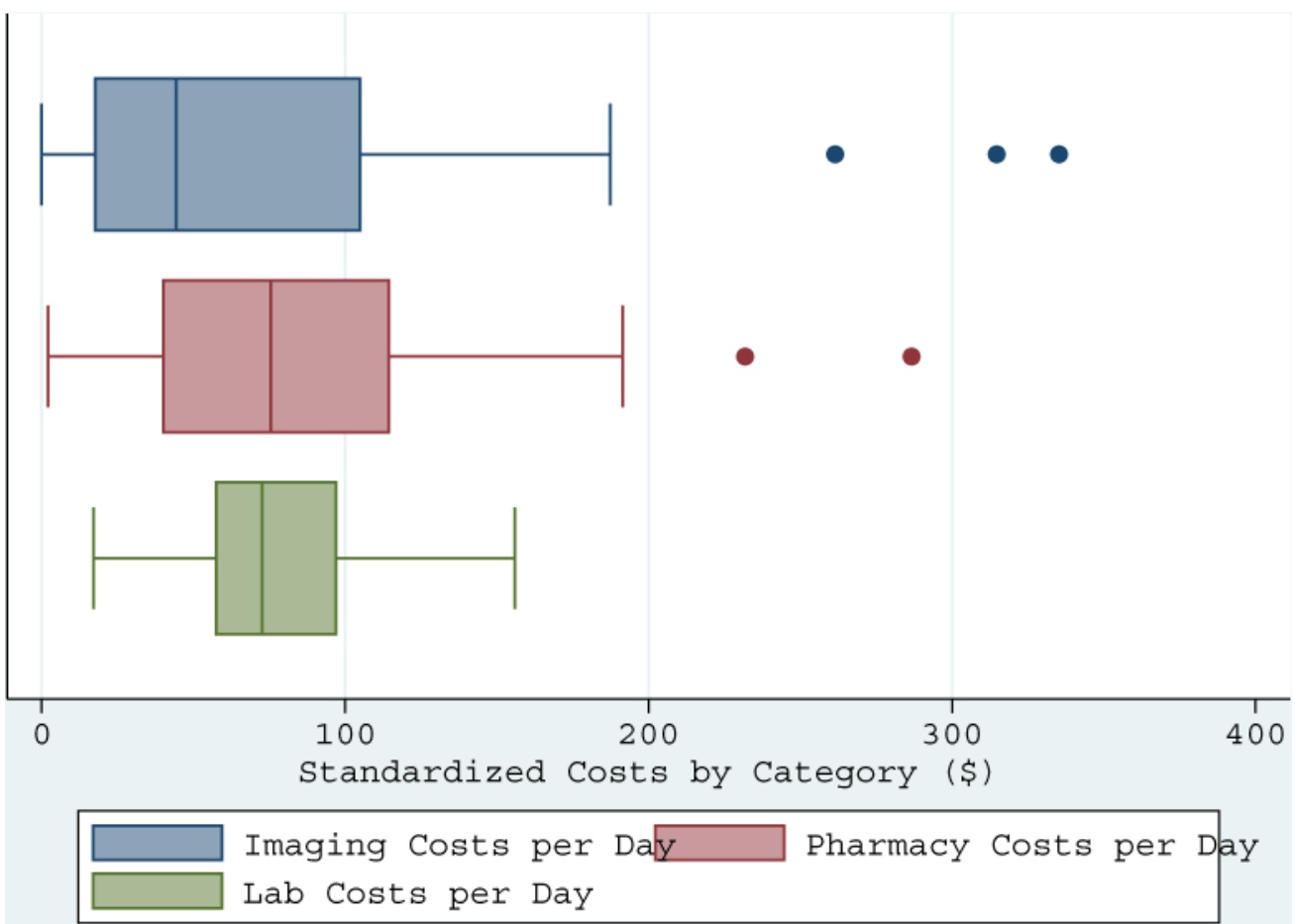

Figure 1.

Distribution of length of stay in cohort. 


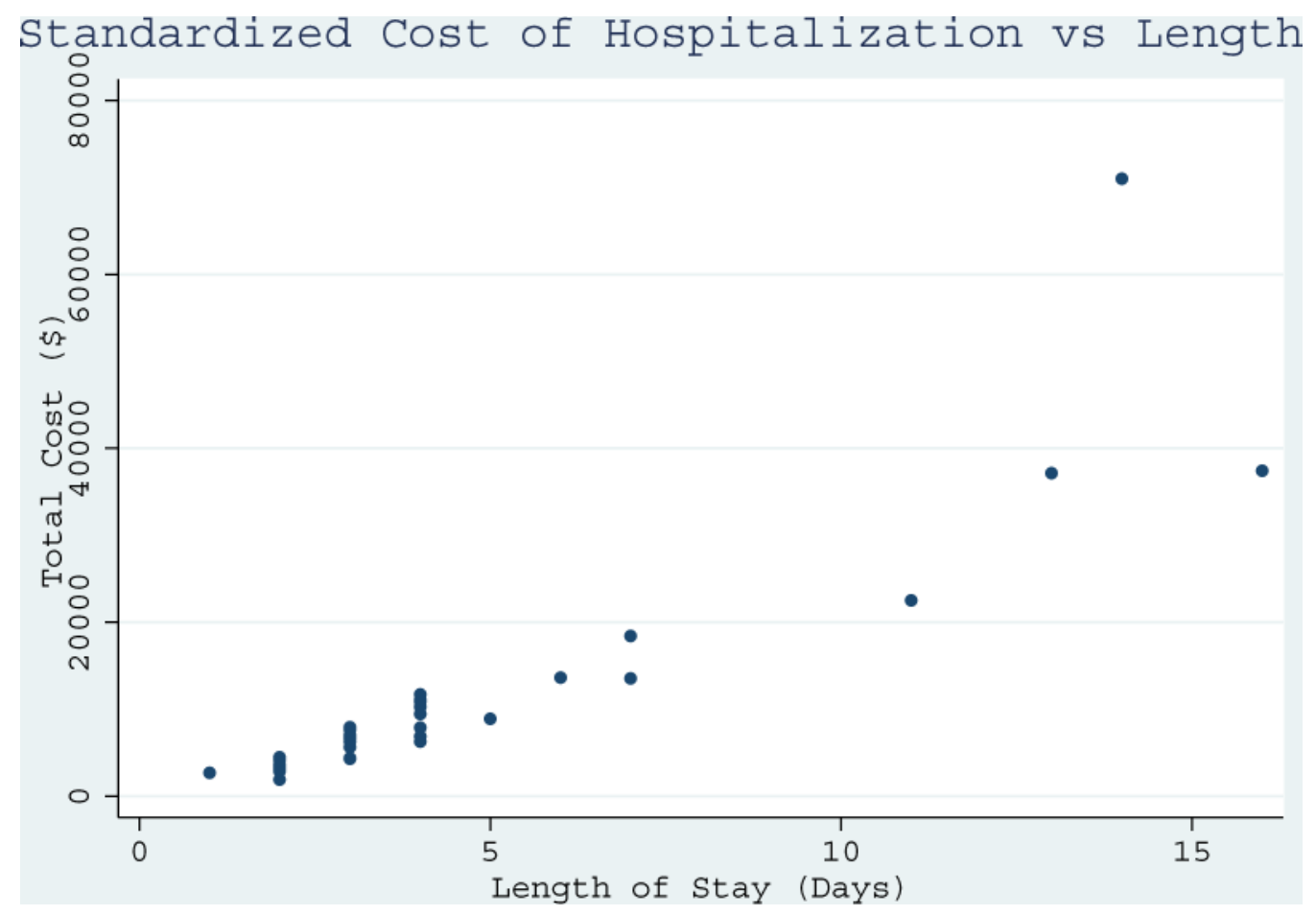

Figure 2.

Total standardized cost of hospitalization vs. length of stay. 


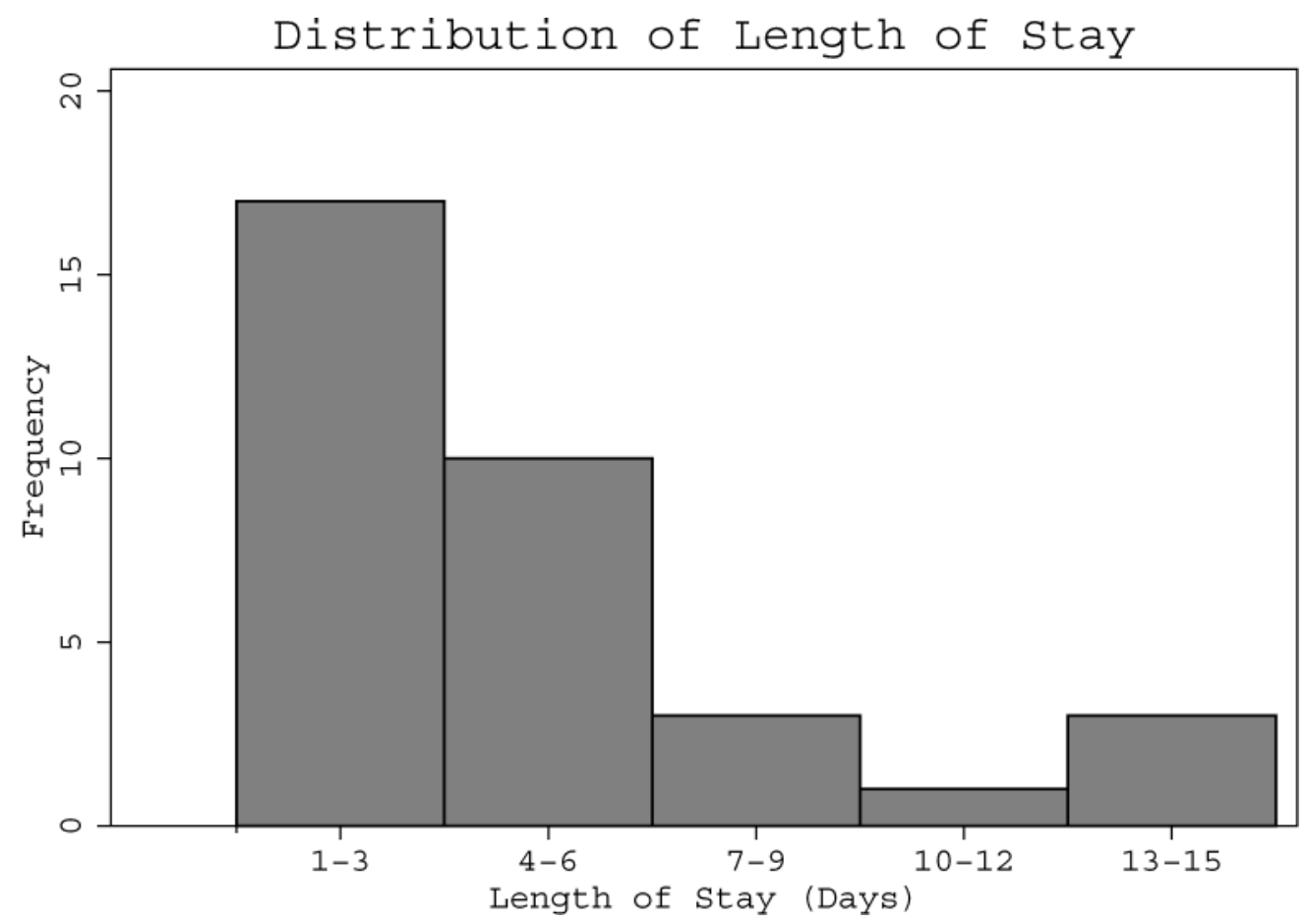

Figure 3.

Standardized costs by category. 
Table 1

Distribution of Standardized Costs for Patients Hospitalized with NLEC (rounded to nearest dollar).

\begin{tabular}{|l|l|l|l|l|}
\hline Category & 25\% Percentile & Median & Mean & 75\% Percentile \\
\hline Total Cost & 4,313 & 7,341 & 11,480 & 11,733 \\
\hline Total Cost/Day & 1,773 & 2,087 & 2,186 & 2,574 \\
\hline Lab Cost/Day & 58 & 73 & 77 & 97 \\
\hline Imaging Cost/Day & 18 & 44 & 74 & 105 \\
\hline Antibiotic Cost/Day & 40 & 75 & 87 & 114 \\
\hline Room and Board/Day & 887 & 975 & 1,045 & 1,243 \\
\hline
\end{tabular}

